\section{Visión Electrónica Más que un estado sólido \\ https://doi.org/10.14483/issn.2248-4728}

UNIVERSIDAD DISTRITAL

FRANCISCO JOSÉ DE CALDAS

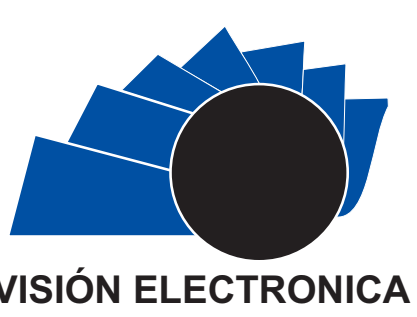

Visión de caso

\title{
Evaluación de métodos de sintonización para modelamiento de controladores
}

\author{
Evaluation of tuning methods for controller modeling
}

Juan Pablo González Penagos ${ }^{1}$, César Augusto Hernández Suarez ${ }^{2}$

\section{INFORMACIÓN DEL ARTICULO}

Historia del articulo

Enviado: 25/01/2018

Recibido: 20/02/2018

Aceptado: 08/06/2018

\section{Palabras clave:}

Error Absoluto, Error Cuadrático, Modelamiento, Sintonización.

\section{Keywords:}
Absolute Error,
Quadratic Error, Modeling,

Tuning

\begin{abstract}
RESUMEN
$\overline{\text { Los métodos de sintonización de controladores para la identificación de modelos de }}$ primer orden más tiempo muerto, de segundo orden sobreamortiguado más tiempo muerto, y de segundo orden subamortiguado más tiempo muerto, pueden expresar errores de predicción absoluto o cuadrático bastante altos. Por lo anterior, en esta investigación se evaluaron tres métodos de sintonización: el método de la tangente modificado de Miller, el método de Smith, y el método de Strejc; luego, mediante MatLab® y su herramienta "System Identification", se realiza la simulación para comparar los métodos expuestos y evaluarlos. Se obtiene que el método más adecuado para la sintonización de controladores es el de Strejc de polos múltiples, debido a su bajo error de predicción absoluto y cuadrático. Se concluye que esta sintonización es una alternativa plausible de modelamiento para un adecuado seguimiento a la función nativa del controlador, así los múltiples polos del controlador presenten respuestas lentas.
\end{abstract}

\footnotetext{
1 Tecnólogo en Electricidad, Universidad Distrital Francisco José de Caldas, Colombia. Lugar de trabajo: Pontificia Universidad Javeriana. Correo electrónico:jpgonzalezp@javeriana.edu.co. ORCID: https://orcid.org/0000-0002-5621-5874.

2 Ingeniero Electrónico, Universidad Distrital Francisco José de Caldas, Colombia. MSc. en Ciencias de la Información y las Comunicaciones, Universidad Distrital Francisco José de Caldas. Ph.D. En Ingeniería, Universidad Nacional de Colombia. Docente Universidad Distrital Francisco José de Caldas, Colombia. Correo electrónico: cahernandezs@correo.udistrital.edu.co. ORCID: https://orcid.org/0000-0001-9409-8341. Citar este artículo como: J. P. González-Penagos y C. A. Hernández-Suarez, "Evaluación de métodos de sintonización para modelamiento de controladores", Visión electrónica, algo más que un estado sólido, vol. 1, no. 1, Edición especial, enero-junio 2018. DOI revista: https://doi.org/10.14483/issn.224.8-4728.
} 


\section{Introducción}

Hoy en día, la apropiada implementación de sistemas de control requiere de una adecuada sintonización del controlador; por ende, se debe garantizar una adecuada selección de sus parámetros, [1]. Los diferentes tipos de función de transferencia usualmente utilizadas para la sintonización de controladores son: sistemas de primer orden más tiempo muerto, sistemas de segundo orden sobramortiguado más tiempo muerto y sistema de segundo orden subamortiguado más tiempo muerto.

De otro lado, la aplicación de los métodos de sintonización es válida dentro de los intervalos para los que han sido desarrollados y conducen a bajar los valores de la ganancia del controlador conforme aumentan los retrasos. Esto conduce a respuestas muy lentas, especialmente en los casos en los que el tiempo muerto es muy grande, [2]. También se ha demostrado que el método de la tangente modificado de Miller, recomendado en la literatura, da un rendimiento pobre tanto a valores pequeños como grandes de tiempo muerto, [3].

En esta investigación, los métodos de sintonización que se procederán a su respectivo análisis son: el método de la tangente modificado de Miller, el método de Smith de dos puntos y por último, el método de Strejc para polos múltiples.

Para su respectiva evaluación se procederá al cálculo del error de predicción absoluto (IEAP) y el error de predicción cuadrático (IECP), para determinar cuál método nos garantiza un modelo cercano al modelo real de la planta, también se empleará MatLab con la opción "System Identification" para obtener directamente un modelo automático y realizar su respectiva comparación

Por lo tanto, el objetivo de este trabajo es realizar una evaluación comparativa de los métodos de sintonización expuestos, para así mediante la comparación del error predictivo absoluto y cuadrático identificar un método adecuado para la sintonización de controladores, para ello se modelará un circuito RC el cual se compone de una fuente de alimentación DC de $12[\mathrm{~V}]$ y un capacitor de $10,31 \mu \mathrm{F}$ en serie con una resistencia de 129,83 $\Omega$.

La estructura del artículo es la siguiente: inicialmente se presentan los materiales y métodos basados en sistemas de transferencia y el indicador de error de cada método y su comparación; posteriormente se realizan las pruebas para ilustrar los resultados obtenidos; y finalmente se presentan las conclusiones.

\section{Materiales y Métodos 2.1. Tipos de Sistemas de Transferencia}

Los métodos para realizar una correcta sintonización del controlador se basan en el modelo reducido de la planta, ya que este permite representar sistemas dinámicos de orden más elevado. Es por lo anterior que, en el momento de identificar una planta, son empleados los modelos matemáticos de primer y segundo orden con tiempo muerto, tal cual se indica en las ecuaciones (1), (2) y (3), [4].

Para el modelo de segundo orden con tiempo muerto se considera la respuesta en bucle abierto del proceso; el estado del proceso puede variar de un sobreamortiguado a un estado críticamente amortiguado, [5].

\section{Primer Orden Más Tiempo Muerto}

$G_{p}(s)=\frac{k_{p} e^{-t_{m} s}}{T s+1}$

Segundo orden sobreamortiguado más tiempo muerto

$G_{p}(s)=\frac{k_{p} e^{-t_{m} s}}{\left(T_{1} s+1\right)\left(T_{2} s+1\right)}$

Segundo orden subamortiguado más tiempo muerto

$G_{p}(s)=\frac{w_{n}^{2} k_{p} e^{-t_{m} s}}{s^{2}+2_{s} w_{n} s+w_{n}^{2}}=\frac{k_{p} e^{-t_{m} s}}{T^{2} s^{2}+2_{s T s}+1}$ 


\subsubsection{Método de la Tangente Modificado de Miller}

Se basa en el método aplicado por Ziegler y Nichols, el cual se considera el primer método propuesto para la sintonización de controladores; se identifica por poseer la característica de que el valor de la respuesta solo tiene un valor finito en un tiempo determinado. El método consiste en trazar una línea tangente a la curva de reacción del proceso en su punto de inflexión o de máxima pendiente, de esta manera poder obtener el tiempo muerto, para la obtención de la constante de tiempo, está comprendida entre la finalización del tiempo muerto y el $63,2 \%$ del cambio total correspondiente a la señal de entrada, [6].

\subsubsection{Método de Smith de Dos Puntos}

El método de dos puntos de Smith fue el primer método propuesto sobre el análisis de dos puntos en la curva característica, el cual toma tiempos pertinentes de $28,3 \%$ y $63,2 \%$ del valor final de la gráfica. Para lograr identificar la planta, inicialmente se requiere la constante de tiempo y el tiempo muerto aparente del sistema, por ende, a partir de dos puntos respectivos de la gráfica se establecen dos ecuaciones con dos incógnitas, que al evaluarlas y solucionarlas matricialmente se obtienen las constantes de tiempo muerto, apareciendo un modelo de primer orden más tiempo muerto, [7].

\subsubsection{Método de la Strejc}

El método de Strejc posee bases del método de la recta tangente; es un caso particular de un método de polos múltiples en el cual las constantes halladas en el sistema son relativamente similares. El método de Strejc siempre posee una caracterización más adecuada para modelos de segundo orden más tiempo muerto, [8].

\subsection{Error Predictivo Absoluto y Cuadrático}

También llamados índices de desempeño. El error predictivo absoluto y el error predictivo cuadrático se calculan a partir de las ecuaciones $(4)$ y $(5)$, donde $y_{p}(t)$ es la respuesta de la planta y $y_{m}(t)$ es la respuesta del modelo identificado según el método utilizado $\left[\begin{array}{lll}1 & 0\end{array}\right]$.

$$
\begin{aligned}
& I E A P=\int_{0}^{\infty}\left|y_{p}(t)-y_{m}(t)\right| d t \\
& I E C P=\int_{0}^{\infty}\left(y_{p}(t)-y_{m}(t)\right)^{2} d t
\end{aligned}
$$

\section{Resultados}

\subsection{Método de la Tangente Modificado de Miller}

Este método aplica el mismo principio del método de Ziegler y Nichols, con la cualidad que la constante de tiempo $\mathcal{\tau}$ se calcula cuando la señal de respuesta del sistema alcanza un valor de $63,2 \%$ del valor total a partir del tiempo muerto. Bajo la definición anterior, se dice que el $63,2 \%$ de $12,0444 \mathrm{~V}$ corresponde a $7,61206 \mathrm{~V}$, obteniendo de esta manera un valor de constante de tiempoigual a $\boldsymbol{\tau}=0,0012237 \mathrm{~s},[7]$.

Para la obtención de la ganancia se realiza el cociente entre $\Delta y$ el cual corresponde a la magnitud de tensión de la señal de respuesta y $\Delta u$ corresponde a la magnitud de tensión de la señal de entrada. Para los parámetros de la ecuación (1), el sistema según el método de la tangente modificada de Miller corresponde a la función de transferencia mostrada en la ecuación (6) y posee el comportamiento visualizado en la Figura 1.

$G_{p}(s)=\frac{1,0119 * \theta^{-8,47 * 10^{-5} * s}}{1,223 * 10^{-8} * s+1}$

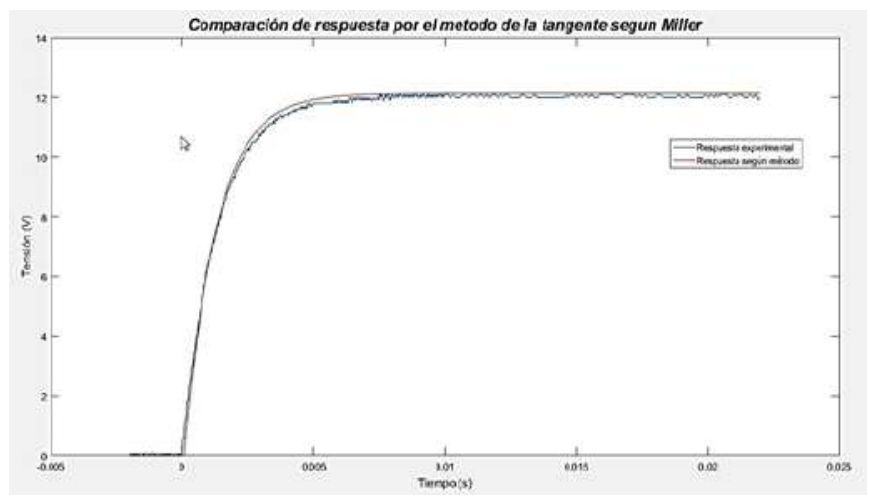

Figura 1. Comparación de la respuesta identificada por el modelo matemático de Miller. Fuente: Elaboración propia.

\subsubsection{Error del Método de la Tangente Modificado de Miller}

Teniendo en cuenta las definiciones de las ecuaciones (4) y (5) se logran determinar el error predictivo absoluto y error predictivo cuadrático en las ecuaciones (7) y (8), respectivamente.

$$
\begin{gathered}
I E A P=0,0029 \\
I E C P=5,2905 * 10^{-4}
\end{gathered}
$$




\subsection{Método de Dos Puntos de Smith}

A partir de los datos de carga del capacitor se establece los puntos del vector tiempo, en los cuales se alcanza el $28,3 \%$ y el $63,2 \%$ del valor final en la señal de respuesta del sistema, estos puntos corresponden a 0,0004 s y 0,00132 s respectivamente, [7].

Con dichos valores a partir del modelo matemático de dos ecuaciones lineales simultáneas con dos incógnitas que corresponde a las ecuaciones (9) y (10), se determina el valor de tiempo muerto $\mathrm{y}_{m}=-0,00004 \mathrm{a}$, correspondiendo a un valor de

$\tau=0,0013$

$$
\begin{aligned}
& t_{28 \%}=t_{m}+\tau / 3 \\
& t_{63 \%}=t_{m}+\tau
\end{aligned}
$$

La solución del sistema de ecuaciones se identifica con las ecuaciones (11) y (12), el cual nos permite caracterizar el modelo matemático de la planta por medio del método de Smith que corresponde a un modelo de primer orden más tiempo de muerto, representado en la ecuación (13). Se logra visualizar la respuesta ante un escalón unitario en la Figura 2. La ganancia se calcula a partir de realizar el cociente del valor final de la señal de paso sobre el valor final de la señal de respuesta del sistema, dando como resultado un valor de $1,0084,[7]$.

$$
\begin{aligned}
& \tau=1,5 *\left(t_{63 \%}-t_{28 \%}\right) \\
& t_{m}=t_{63 \%}-\tau \\
& G p(s)=\frac{(1,0084) * t^{t(0,00004) s}}{(0,0013) s+1}
\end{aligned}
$$

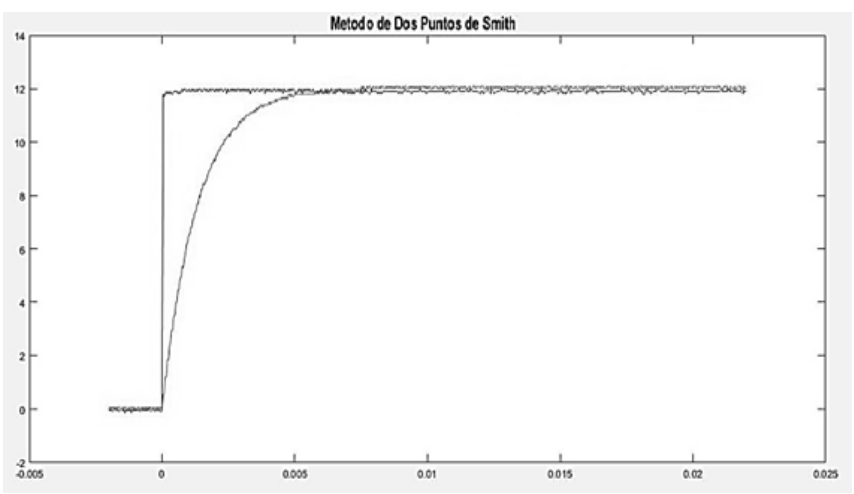

Figura 2. Respuesta del modelo encontrado ante una señal tipo pasó. Fuente: Elaboración propia.

\subsubsection{Error del Método de Dos Puntos de Smith}

Se realiza el cálculo con el fin de determinar el error de predicción absoluto (IEAP) y el error de predicción cuadrático (IECP), los cuales se expresan en las ecuaciones (14) y (15).

IEAP $=0,0014$

IECP $=1,082 * 10^{-4}$

\subsection{Método de Strejc}

Al implementar el Método de Strejc para la sintonización del controlador, se obtendrá un modelo de polos múltiples el cual se puede visualizar en la ecuación (16), para valores grandes de $n$, el sistema se comporta con tiempos muertos largos, [4].

$G_{p}(s)=\frac{k_{p} e^{-t_{m} s}}{(\tau s+1)^{n}}$

Para realizar el modelo respectivo se obtiene los valores de $T_{u}$ y $T_{a}$. Analizando la gráfica se obtiene el cual representa el tiempo muerto, $T_{a}=0,000745 \mathrm{~s}$ representa el tiempo del Método de la Tangente ya mencionado; posteriormente se realiza el cociente entre $T_{u}$ y $T_{a}$ para determinar el orden del modelo $n$ respecto a la Tabla $1,[8]$.

\begin{tabular}{|l|l|l|l|}
\hline $\mathrm{N}$ & $T_{u} / T_{a}$ & $T_{u} / \tau$ & $T_{a} / \tau$ \\
\hline 1 & 0,000 & 0,000 & 0,000 \\
\hline 2 & 0,104 & 0,282 & 2,718 \\
\hline 3 & 0,218 & 0,805 & 3,695 \\
\hline 4 & 0,319 & 1,425 & 4,465 \\
\hline 5 & 0,410 & 2,100 & 5 \\
\hline 6 & 0,493 & 2,811 & 5,699 \\
\hline 7 & 0,570 & 3,549 & 6,226 \\
\hline 8 & 0,642 & 4,307 & 6,711 \\
\hline 9 & 0,000 & 5,081 & 7,164 \\
\hline 10 & 0,773 & 5,869 & 7,590 \\
\hline
\end{tabular}

Tabla 1. Método de Strejc [1]. 
$\mathrm{Al}$ obtener un valor de 0,3154 , se procede a aproximar por debajo, por ende, se obtiene $\mathrm{N}=3$ consecutivamente obtenemos un valor de $T_{V} / \tau=0,805 \mathrm{y} \quad T_{a} / \tau=3,695 \quad$ para proceder a identificar el valor de $\tau$ en las ecuaciones (17) y (18).

$$
\begin{gathered}
\tau_{1}=\frac{T_{u}}{0,805}=2,9193 * 10^{-4}[s] \\
\tau_{2}=\frac{\tau_{a}}{3,695}=2,0162 * 10^{-4}[s]
\end{gathered}
$$

Por lo cual, al obtener un $T_{1}$ y $T_{2}$ comparables, se avala la idoneidad para un modelo de polos múltiples. Se obtiene un promedio de $T_{1}$ y $T_{2}$ para hallar el $\tau$ del modelo, el cual se identifica en la ecuación (19), [1].

$\tau=\frac{\tau_{1}+\tau_{2}}{2}=2,4677 * 10^{-4}[s]$

Al obtener $T_{u}$, el cual caracteriza al tiempo muerto $T_{m}$ se hallan nuevamente los $T_{u}$ referenciados en la Tabla (1), los cuales se nominan como $T_{u t}$ ver ecuación(20).

$T_{u t}=\frac{n * T_{a}}{10}-1=2,2350 * 10^{-4}[s]$

Por lo cual, el tiempo muer to del sistema está dado por la ecuación(21):

$$
T_{m}=T_{u}-T_{u t}=1,1500 * 10^{-5}[s]
$$

Finalmente se obtiene un modelo de polos múltiples expresado en la ecuación(22):

$$
G_{p}(s)=\frac{1,0119 e^{1,1500 * 10^{-5} s}}{\left(2,4677 * 10^{-4} s+1\right)^{3}}
$$

\subsubsection{Error del Método de Strejc}

Se realiza el cálculo con el fin de determinar el error de predicción absoluto (IEAP) y el error de predicción cuadrático (IECP), los cuales se expresan en la ecuación (23) y (24).

$I E A P=0,0009$

$I E C P=0,712 * 10^{-4}$

3.4. Identificación de la Planta por Medio de "System Identification" - MatLab
Para la identificación de la planta de forma automática mediante el software Matlab [9] se tienen en cuenta los parámetros iníciales de la obtención de puntos tanto de la señal de entrada como de la salida. De esta forma, descartando los tiempos negativos obtenidos por el equipo de medida y los puntos reiterados, se obtienen tiempos de muestreo de $4 * 10^{-5} s$ el Toolbox de MatLab identifica la señal de entrada con respecto a la señal de salida como se muestra en la Figura 3.
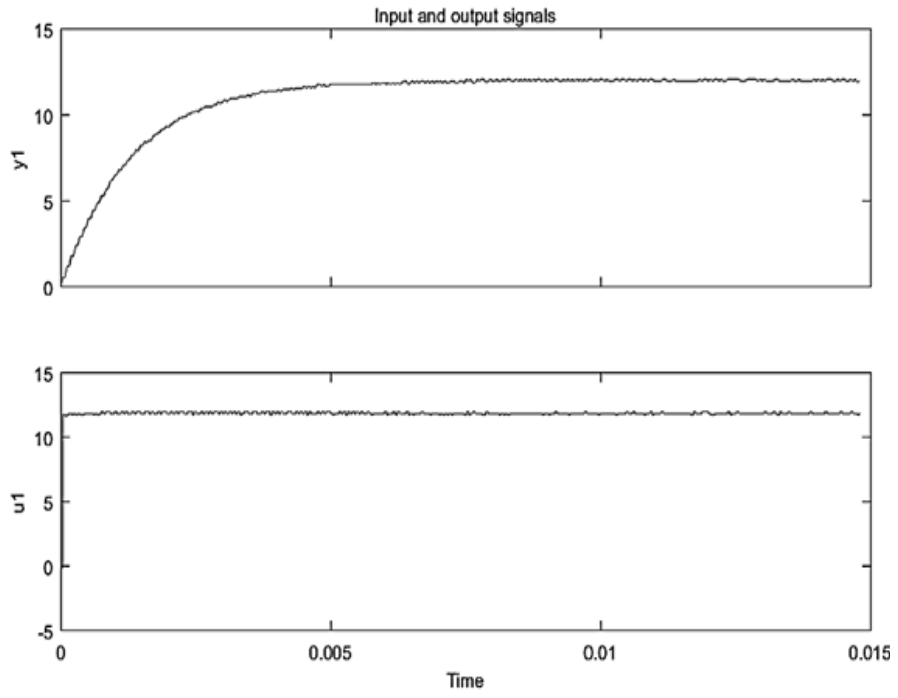

Figura 3. Identificación de la señal de entrada con respecto a la señal de salida de la planta.

Fuente: Elaboración propia.

$\mathrm{Al}$ identificar las señales de entrada y salida de forma experimental, se prueban diferentes funciones de transferencia con ceros y polos adicionales al sistema, así como retardos en dicha función. La respuesta de las funciones de transferencia obtenidas para la misma planta se describe en la Figura 4.

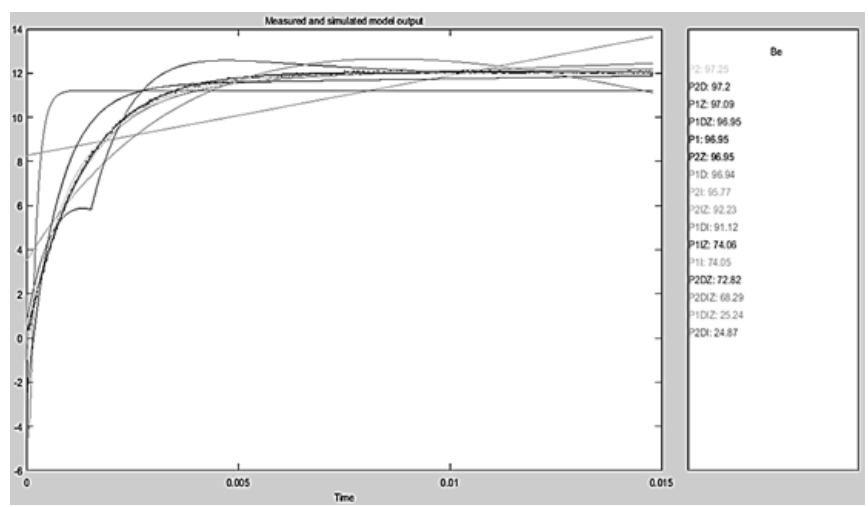

Figura 4. Respuesta de las funciones de trasferencia identificadas por System Identification.

Fuente: Elaboración propia 
Al visualizar la Figura 4 se identifican las tres primeras funciones de transferencia obtenidas, las cuales poseen un factor de certeza del 97,25\%, 97,2\% y $97,09 \%$ respectivamente. Por lo cual, el mejor modelo matemático identificado por el "System Identification" corresponde a la función de transferencia de segundo orden sin retardo, ya que posee un factor de certeza del 97,25\%, la cual se estructura en la ecuación (25), [9].

$G_{p}(s)=\frac{1,0145}{(1+168,36 * S) *(1+32,57 * S)}$

\subsection{Evaluación Comparativa de Error de Predicción Absoluto y Cuadrático}

El análisis y comparación de los errores de predicción absoluto y cuadrático de los métodos evidenciados, se expresan en la Tabla (2).

\begin{tabular}{|c|c|c|c|}
\hline MÉTODO & MODELO & IEAP & IECP \\
\hline $\begin{array}{c}\text { Método de la tangente } \\
\text { modificado por Miller }\end{array}$ & $G_{P} S=\frac{1,00119 * \mathrm{e}^{-0,000235 * \mathrm{~s}}}{0,001085 * \mathrm{~s}+1}$ & 0,0029 & $\begin{array}{l}5,2905 \\
* 10^{-4}\end{array}$ \\
\hline $\begin{array}{c}\text { Método de dos puntos } \\
\text { de Smith }\end{array}$ & $\mathrm{G}_{\mathrm{P}} \mathrm{S}=\frac{(1,0084) * \mathrm{e}^{\mathrm{t}(0,00004)^{\mathrm{s}}}}{(0,0013)^{\mathrm{s}}+1}$ & 0,0014 & $1,082 * 10^{-4}$ \\
\hline Método de Strejc & $\mathrm{G}_{\mathrm{P}} \mathrm{S}=\frac{1,0119 \mathrm{e}^{1,1500 * 10^{-5} \mathrm{~s}}}{\left(2,4677 * 10^{-4} \mathrm{~s}+1\right)^{3}}$ & 0,0009 & $0,712 * 10^{-4}$ \\
\hline
\end{tabular}

Tabla 2. Comparación Métodos de Sintonización Respectivos. Fuente: Elaboración propia

Se logra identificar que el modelo que mejor representa el controlador de estudio es el de Strejc de polos múltiples, debido a que tiene un menor error de predicción absoluto (IEAP) y también un menor error de predicción cuadrático (IECP).

\section{Conclusiones}

El método de Smith obtuvo un resultado intermedio en cuanto a la representación de la planta como se observa en el error IEAP, por tanto, se puede concluir que es una alternativa aceptable al sintonizar controladores, al caracterizarse por ser una función de transferencia de primer orden y su perfil de tratamiento de la información no posee gran complejidad.

Una característica de los modelos que representaron de mejor forma el comportamiento de la respuesta de la planta, al comparar los errores IEAP e IECP, es el hecho de tener polos muy alejados del origen del plano S, esta característica identifica una respuesta lenta del sistema.
La herramienta del Software MatLab "System Identification" es un método rápido y sencillo de identificación del modelo matemático de una planta experimental, como también una gran ventaja en la obtención de la función de transferencia, que se puede acomodar al objetivo a analizar, por ello, se obtuvo una función de transferencia de segundo orden sin retardo con un error de predicción absoluto IEAP $=3,4^{*} 10^{-6}$ y un error de predicción cuadrático de IECP $=2,8 * 10^{-4}$

El método de Strejc establece un modelo de polos múltiples caracterizándose por superar los modelos del método de dos puntos de Smith y el método de la tangente modificada de Miller, debido a que posee un menor IEAP y IECP que los demás métodos, uno de los grandes inconvenientes del modelo de Strejc es la localización de los polos, debido a que son muy alejados del plano S, por lo cual el sistema posee un comportamiento lento. 


\section{Referencias}

[1] V.Alfaro, "Identificación de modelos de orden reducido a partir de la curva de reacción del proceso", Ciencia y tecnología, vol. 24, no. $2,2006$.

[2] M.A. Rodrigo, "Control de procesos con dinámica compleja”, España, 2012.

[3] W. L. Luyben, "Tuning proportionalintegral controllers for processes with both inverse response and deadtime", Industrial $\mathcal{E}^{\circ}$ engineering chemistry research, vol. 39, no. 4, 2000, pp. 973-976.

[4] K. J. Åström, T. Hägglund, "Benchmark Systems for PID Control”, IFAC Proceedings Volumes, vol. 33, no. 4, 2000, pp. $165-66$. DO I :

https://doi.org/10.1016/S1474$6670(17) 38238-1$

[5] A. Jahanmiri y H. Fallahi, "New methods for process identification and design of feedback controller", Chemical Engineering Research and Design, vol. 75, no. 5, 1997, pp. 519-522.

[6] Miller J., Department of Chemical Engineering Report, Louisiana State University, Baton Rouge, Louisiana, Estados Unidos, 1967.

[7] C. L. Smith, "Digital computer process control”, Intext Educational Publishers, 1972.

[8] V. Strejc, C. Glatzel, "Apuntes del curso EL633 - Control e Instrumentación Industrial", Departamento de Electricidad, Universidad de Chile, Santiago, Chile, 1974.

[9] MATLAB and Statistics Toolbox Release 2012b, The MathWorks, Inc., Natick, Massachusetts, United States.

[10] M. Verdugo, M. Cal y C. Fernandez, "Análisis econométrico: Una aproximación practica con shazam”, Delta Publicaciones, 2009. 